\title{
ASPECTOS TAXONÓMICOS Y DE CONSERVACIÓN DE Melocactus peruvianus Vaupel Y Neoraimondia arequipensis subsp. roseiflora (Werderm. \& Backeb.) Ostolaza EN EL VALLE DEL RÍO CHILLON, LIMA: CERRO UMARCATA Y QUEBRADA OROBEL
}

\section{TAXONOMIC AND ECOLOGICAL ASPECTS OF Melocactus peruvianus Vaupel AND Neoraimondia arequipensis subsp. roseiflora (Werderm. \& Backeb.) Ostolaza AT THE CHILLÓN RIVER VALLEY: UMARCATA HILL AND OROBEL VALLEY}

\author{
Viviana Castro-Cepero $^{1}$ y Aldo Ceroni-Stuva ${ }^{2}$
}

\begin{abstract}
Resumen
Se presenta el estudio de la taxonomía y estado de conservación de dos cactáceas, Melocactus peruvianus Vaupel y Neoraimondia arequipensis subsp. roseiflora (Werderm. \& Backeb.) Ostolaza, ubicadas en el cerro Umarcata y la quebrada Orobel, en la cuenca baja del río Chillón. Las plantas fueron recolectadas en los meses de febrero y julio de 2003 y febrero, mayo, agosto y diciembre de 2004, registrándose datos sobre las características taxonómicas, así como de ubicación y estado de conservación. Melocactus peruvianus, cactus globoso y solitario, de 7 a 17 cm de alto, distribuido desde la frontera entre Perú y Ecuador hasta Arequipa (Perú), se encuentra en estado Vulnerable (VU), mientras que Neoraimondia arequipensis subsp. roseiflora, cactus columnar, robusto que alcanza de 1.3 a $4 \mathrm{~m}$ de alto, endémico de Perú, distribuido en los departamentos de Ancash, Lima e Ica, se encuentra en estado de Preocupación Menor (LC).

Palabras clave: taxomomía, conservación, Cactaceae, cactus, Chillón
\end{abstract}

\begin{abstract}
We present the study on the taxonomy and conservation status of two cacti, Melocactus peruvianus Vaupel and Neoraimondia arequipensis subsp. roseiflora (Werderm. \& Backeb.) Ostolaza, from Umarcata Hill and Orobel Valley, in the lower river basin of the Chillon River. Plants were collected in February and July 2003, and February, May, August, and December 2004, recording data on taxonomic characteristics, as well as location and condition. Melocactus peruvianus is a solitary globose cactus, from 7 to $17 \mathrm{~cm}$ tall, distributed from the borderline between Peru and Ecuador to Arequipa in Peru, and its conservation status is Vulnerable (VU). Neoraimondia arequipensis subsp. roseiflora is a columnar cactus, from 1.3 to $4 \mathrm{~m}$ tall, endemic to Peru, distributed in the departments of Ancash, Lima and Ica. Its conservation status is Least Concern (LC).
\end{abstract}

Key words: taxonomy, conservation, Cactaceae, cacti, Chillon

\section{Introducción.}

La familia Cactaceae en el Perú está representada por 34 géneros y más de 255 especies (Hunt, 1999). Estas plantas están distribuidas en casi todos los ecosistemas, desde los desiertos costeros, vertiente occidental, puna, valles interandinos y llegando exitosamente al bosque tropical amazónico (Calderón et al., 2004). Las cactáceas conforman los elementos característicos en la formación vegetal conocida como Piso de Cactáceas Columnares, formación xerofítica ubicada al centro y sur de la vertiente occidental del Perú, dominada por arbustos y cactáceas columnares (Weberbauer, 1945). Dentro de los ecosistemas áridos en Lima, el cerro Umarcata y la quebrada Orobel se presentan como zonas de alta diversidad específica de cactáceas (Teixeira et al., 2004). Estudios realizados en estas zonas en cuanto a la taxonomía, diversidad y densidad de las cactáceas (Castro, 2006; Teixeira et al., 2004), se resalta el rol de una de las especies dominantes como es Neoraimondia arequipensis subsp. roseiflora; a través del estudio de su fenología y su relación con la hormiga Camponotus sp., (Novoa et al., 2005a; 2005b y 2005c), así como de conservación ex situ de cactáceas de Lima (Ceroni et al., 2004), demuestran que estas zonas son áreas importantes a conservar no sólo por ser lugares de una alta diversidad de estas especies, sino también por los futuros trabajos de investigación que puedan hacerse a fin de entender más su dinámica para una mejor gestión de los ecosistemas áridos del Perú (Ceroni \& Castro, 2006). Las especies de cactáceas que se pueden encontrar en estos lugares son un total de 9 pertenecientes a 8 géneros y 2 subfamilias (Castro, 2006; Calderón et al., 2007). En la subfamilia 
Cactoidea: Neoraimondia arequipensis subsp. roseiflora; Espostoa melanostele; Haageocereus pseudomelanostele subsp. aureispinus; Haageocereus acranthus subsp. backebergii; Cleistocactus acanthurus subsp. faustianus; Melocactus peruvianus y Mila nealeana; mientras que en la subfamilia Opuntioideae: Austrocylindropuntia pachypus y Cumulopuntia sphaerica. En la presente nota científica se presenta algunos aspectos taxonómicos y estado de conservación de dos de estas especies de la subfamilia Cactoideae, Melocactus peruvianus Vaupel y Neoraimondia arequipensis subsp. roseiflora (Werderm. \& Backeb.) Ostolaza, pertenecientes a las Tribus Cereeae y Browningieae, respectivamente.

\section{Materiales y métodos.}

Área de estudio.

El piso de Cactáceas columnares se encuentra ubicado entre los 1100 y 2400 msnm. La primera zona de colecta, llamada Cerro Umarcata, se encuentra a una distancia de 6 kilómetros del centro del distrito de Santa Rosa de Quives, a la altura del kilómetro 69 de la carretera Lima - Canta, a una altitud de 1260

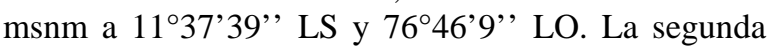

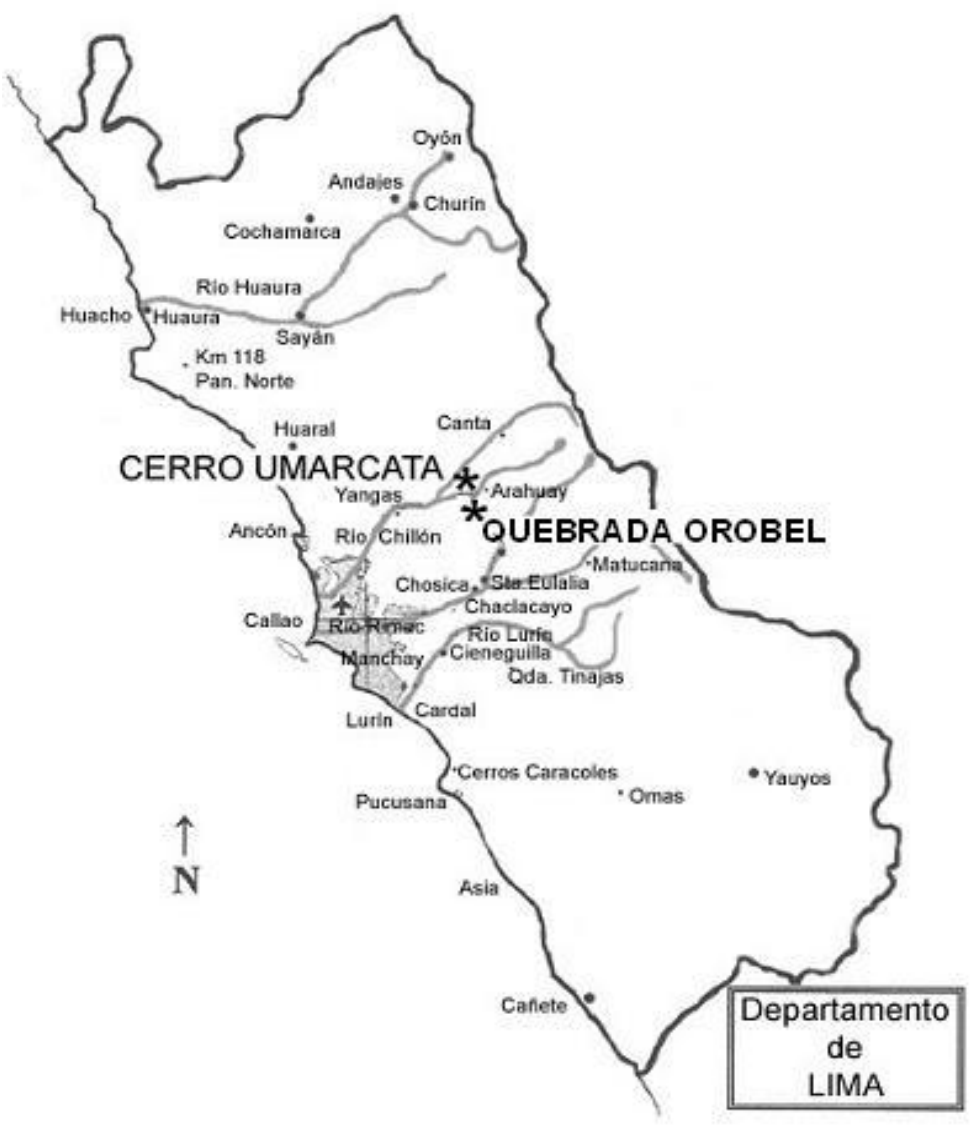

Figura 1. Ubicación de las zonas de colecta en el área de estudio. zona de colecta, llamada Quebrada Orobel, se encuentra a una distancia de 4 kilómetros de Quives, a la altura del kilómetro 4 de la carretera a Arahuay, a

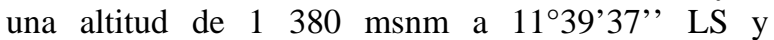
7645'26', LO (Figura 1). El análisis de suelo realizado en el Laboratorio de Análisis de Suelos y Plantas de la UNALM, permite caracterizarlo con un pH que varía entre 5 y 7.6; la conductividad eléctrica varía entre 0.69 y $1.96 \mathrm{dS} / \mathrm{m}$; el porcentaje de carbonato de calcio alcanza un valor máximo de 1.3; el porcentaje de materia orgánica varía entre 1.3 y 3.6; el contenido de fósforo oscila entre 22.2 y 41.3 ppm y el contenido de potasio, entre 340 y 860 ppm.

Colección botánica.

Se colectaron las especies de cactáceas presentes en el Cerro Umarcata y Quebrada Orobel, distrito de Santa Rosa de Quives, en el Valle del Río Chillón, registrándose los datos de cada individuo en las etiquetas y libretas de campo, así como datos de ubicación entre otros. Las plantas fueron recolectadas al azar en los meses de febrero y julio de 2003 y febrero, mayo, agosto y diciembre de 2004. Se colectó casi toda la planta haciendo un corte en la parte basal. Las flores y frutos se recolectaron en frascos o sobres, identificadas apropiadamente $y$ tratadas en el gabinete. Las plantas fueron depositadas en el Herbario de Sitio del Jardín Botánico "Octavio Velarde Núñez" de la Universidad Nacional Agraria La Molina. Estudio taxonómico.

Las muestras colectadas fueron tratadas en el Centro de Interpretación "Casa Julio Gaudron” del Jardín Botánico “Octavio Velarde Núñez” de la Universidad Nacional Agraria La Molina. Se cortó el espécimen de la base al ápice, aislando las costillas, en secciones longitudinales y transversales. Todas las muestras tomadas se llevaron al secador en una prensa convencional, cambiando el papel con frecuencia y utilizando para la preservación, solución FAA y bórax (fungicida). Los frutos se prensaron aparte y fueron cortados en secciones longitudinales y transversales. Antes de seccionar el material se registraron las dimensiones de cada individuo. El material fértil sirvió para la determinación taxonómica del material recolectado (flores, frutos y semillas) $\mathrm{y}$ fue preservado en frascos con alcohol o congelado, hasta su uso. La determinación taxonómica se realizó en base a claves de determinación y en base a la comparación con muestras conservadas en los herbarios de la 
Universidad Nacional Agraria La Molina (MOL) y Universidad Nacional Mayor de San Marcos (UNMSM).

\section{Resultados y discusión.}

Subfamilia Cactoideae Buxbaum.

Plantas arborescentes, arbustivas, cespitosas, trepadoras o epífitas; raíces fibrosas o pivotantes; tallos ramificados desde la base, parte media o ápice, no segmentados, globosos o columnares, con costillas y tubérculos; zonas reproductivas no diferenciadas o diferenciadas en cefalios; hojas vestigiales o ausentes; gloquidios ausentes; flores sésiles, diurnas o nocturnas; pericarpelo desnudo o con escamas y areolas; tubo floral corto o elongado; areolas florales con pelos, espinas o cerdas; estambres numerosos insertos en el tubo del perianto; frutos secos o carnosos, de tamaño y forma variable; semillas ovaladas o circulares a veces con expansiones y testa de arquitectura variada; arilo ausente (Anderson, 2001; Barthlott \& Hunt, 1993; Hunt et al., 2006). Las tribus Trichocereeae, Brownngieae y Cereeae, que se encuentran en el lugar de estudio, están estrechamente relacionadas a nivel genético (Wallace \& Gibson, 2002) y conforman el clado "BCT". De acuerdo a Taylor \& Zappi (2004), considerando delimitaciones a nivel morfológico, Trichocereeae está aparentemente más relacionado a Cereeae que a Browningieae, donde Cereeae presenta flores desnudas y Trichocereeae presenta flores cubiertas de pelos, espinas y cerdas. Tribu Cereeae Salm-Dyck.

Plantas arborescentes o arbustivas a veces trepadoras; tallos no segmentados, globosos o columnares, con costillas; zonas reproductivas diferenciadas en cefalios laterales o apicales; flores actinomorfas sésiles, diurnas o nocturnas; pericarpelo desnudo o con escamas; frutos carnosos, con perianto persistente; semillas ovaladas sin expansiones (Anderson, 2001; Barthlott \& Hunt, 1993).

Melocactus Link \& Otto. Verhandlungen des Vereins zur Beförderung des Gartenbaues in den Königlich Preussischen Staaten 3: 417.1827 (nomen conservandum).

Planta solitaria, globosa o cilíndrica, no ramificada de crecimiento definido. Costillas marcadas, 8 a 27, verticales, sin tubérculos. Areolas pequeñas a grandes, con 3 a 20 o más espinas muy desarrolladas, a veces curvas. Cefalio apical formado por pelos lanosos blancos y cerdas. Flores pequeñas, tubulares, rojas a rosadas, sin pelos o espinas en el tubo, insertas en el cefalio, estambres de filamento corto epipétalos, ovario ínfero. Fruto corto a largamente aovado, jugoso, sin pelos o espinas, blanco, rojo, rojizo o rojo grosella. Semillas globosas a aovadas. Distribución: Antillas, Brasil, Bahamas, Colombia, Costa Rica, Cuba, República Dominicana, Ecuador, Guatemala, Guyana, Honduras, Haití, Jamaica, México, Nicaragua, Panamá, Perú, Puerto Rico, Surinam,
Trinidad y Tobago y Venezuela (Hunt et al., 2006). En la zona de estudio se determinó una especie.

Melocactus peruvianus Vaupel. Botanische Jahrbücher für Systematik, Pflanzengeschichte und Pflanzengeographie 50: (2-3, Beibl. 111): 28. 1913.

Sinónimos: Cactus townsendii Britton \& Rose. Melocactus amstutziae Rauh \& Backeb. $M$. fortalezensis Rauh \& Backeb. M. huallancensis Rauh \& Backeb. M. jansenianus (Borg) Backeb. $M$. peruvianus var. amstutziae (Rauh \& Backeb.) Rauh \& Backeb. M. peruvianus var. canetensis Rauh \& Backeb. M. peruvianus var. lurinensis Rauh \& Backeb. M. trujillensis Rauh \& Backeb. M. trujillensis var. schoenii Rauh \& Backeb. M. unguispinus Rauh \& Backeb.

Planta globosa solitaria, de 7 a $17 \mathrm{~cm}$ de alto. Tallo terete de 13.5 a $17.5 \mathrm{~cm}$ de diámetro, con 10 a 15 costillas, epidermis visible y verde glauco; areolas redondas a aovadas, de $1 \mathrm{~cm}$ de diámetro mayor, 0.5 $\mathrm{cm}$ de diámetro menor y de $0.1 \mathrm{a} 0.4 \mathrm{~cm}$ de espesor, color marrón; espinas radiales y centrales curvas, 5 a 15 espinas por areola, de 2.3 a $3 \mathrm{~cm}$ de longitud, 0.05 a $0.1 \mathrm{~cm}$ de grosor, de color marrón, amarillo o gris; cefalio apical de 5 a $15 \mathrm{~cm}$ de longitud y 8 a $10 \mathrm{~cm}$ de diámetro, con pelos lanoso blancos y cerdas blancas y rojas; flores actinomorfas, tubulares, rosadas a rojo grosella, desarrollándose en el cefalio apical, entre 2.3 a $3 \mathrm{~cm}$ de longitud y perianto de 0.5 a 1 de diámetro, con aproximadamente 15 tépalos: sepaloide, lanceolado-apiculado, de 0.3 a $0.6 \mathrm{~cm}$ de longitud y de 0.1 a $0.2 \mathrm{~cm}$ de ancho, y petaloide ligulado-apiculado, de 0.5 a $0.6 \mathrm{~cm}$ longitud y de 0.075 a $0.1 \mathrm{~cm}$ de ancho; tubo del receptáculo, de $0.5 \mathrm{~cm}$ de longitud y de 0.3 a $0.4 \mathrm{~cm}$ de diámetro; cámara nectarial de 0.2 a $0.4 \mathrm{~cm}$ de longitud y $0.2 \mathrm{~cm}$ de diámetro; androceo con aproximadamente 90 estambres epipétalos, entre 0.2 y $0.4 \mathrm{~cm}$ de longitud, anteras bitécicas de $0.1 \mathrm{~cm}$ de longitud y $0.05 \mathrm{~cm}$ de diámetro, cremosas, filamentos entre 0.1 y $0.3 \mathrm{~cm}$ de longitud y $0.01 \mathrm{~cm}$ de diámetro, color marfil; pericarpelo de $0.55 \mathrm{~cm}$ de longitud y 0.3 cm de diámetro; gineceo sincárpico de 1.15 a 2 cm de longitud, ovario ínfero de 0.2 a $0.3 \mathrm{~cm}$ de longitud y entre 0.15 a $0.2 \mathrm{~cm}$ de diámetro, 5 carpelos, numerosos óvulos en placentación parietal, estilo de 0.45 a $1.2 \mathrm{~cm}$ de longitud y $0.05 \mathrm{~cm}$ de diámetro, estigma lobulado de 0.15 a $0.25 \mathrm{~cm}$ de longitud y 0.1 cm de diámetro, 5 lóbulos color verde; fruto baya, de color rojo, rojo grosella, elipsoidal alargado de 1.5 a $1.8 \mathrm{~cm}$ de longitud, de 0.6 a $0.7 \mathrm{~cm}$ de diámetro mayor y de 0.3 a $0.4 \mathrm{~cm}$ de diámetro menor, funículo ariloso blanco desarrollado, flor persistente de 1 a $1.2 \mathrm{~cm}$ de longitud (Figuras 2 y 3). Según Ramírez \& Ceroni (2009) las semillas son de forma ovalado-reniforme, negras o marrones, en número moderado (60 a 120), de $1.15 \mathrm{~mm}$ de longitud y $0.88 \mathrm{~mm}$ de diámetro, testa rugosa, región hilo-micropilar grande y oblicua con respecto al eje mayor de la semilla, micrópilo e hilo pequeños. La especie se distribuye desde la frontera 

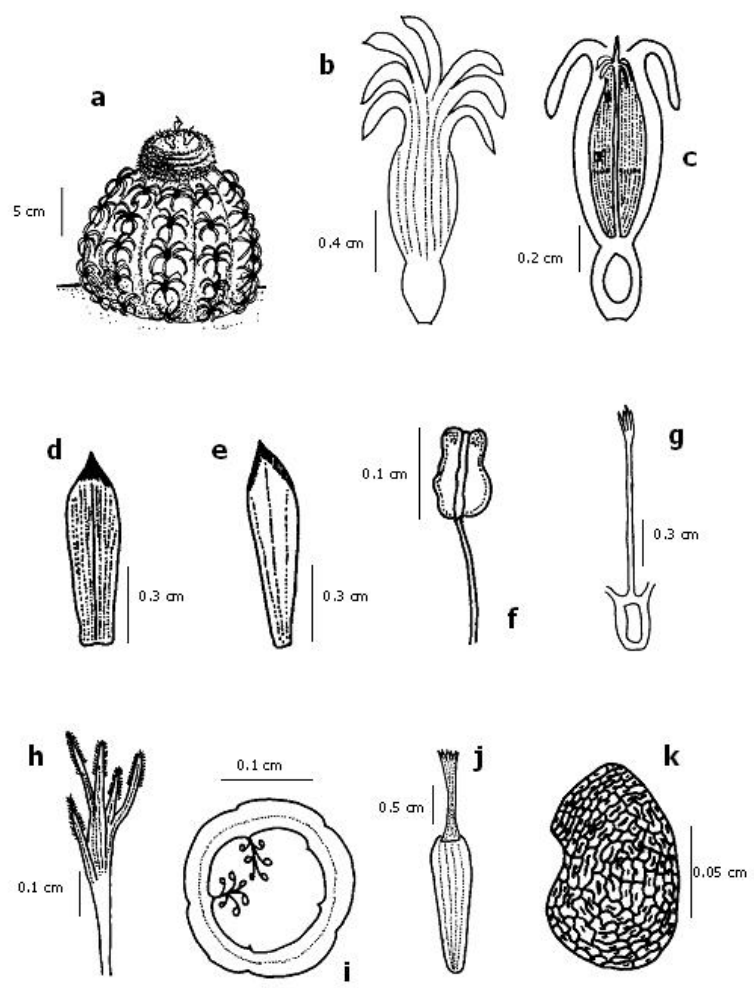

Figura 2. Melocactus peruvianus Vaupel. a. Hábito; b. Flor; c. Sección longitudinal de la flor; d. Tépalo sepaloide; e. Tépalo petaloide; f. Estambre; g. Gineceo; h. Estigma; i. Sección transversal del ovario; j. Fruto; k. Semilla.

entre Perú y Ecuador hasta Perú, donde abarca desde Tumbes, Piura, Lambayeque, La Libertad, Ancash (Santa, Huaylas y Corongo), Lima y Arequipa (Cotahuasi), a una altura de entre 30 y $2000 \mathrm{msnm}$ (Lozada-Castillo, 2009; Ostolaza, 2007).

Material estudiado: LIMA: Cerro Umarcata, Santa Rosa de Quives, agosto de 2004, 1260 msnm, Viviana Castro 117 (Herbario de Sitio Jardín Botánico OVN); Cerro Umarcata, Santa Rosa de Quives, diciembre de 2004, 1260 msnm, Viviana Castro 118 (Herbario de Sitio Jardín Botánico OVN).

De acuerdo a Britton \& Rose (1919 - 1922) el género era conocido en Europa en 1605 como Echinomelocactus, el cual se atribuye a Clusius; luego en 1701 Tournefort lo acortó a Melocactus y por último Linnaeus lo nominó Cactus en 1753.

Anderson (2001) indica que Linnaeus incluyó todas las especies de cactáceas conocidas hasta 1753 en el género Cactus, el cual se constituyó en el género tipo de la familia. Sin embargo de acuerdo a los principios del Código Internacional de Nomenclatura Botánica, debe usarse el nombre más antiguo publicado efectivamente, por lo que el género Mammillaria Haworth 1812 (tipo usado por Jussieu para la descripción de la familia en 1789) se conserva frente a Cactus, el cual se constituye en un género no válido. En 1827 Link \& Otto publicaron efectivamente el género Melocactus.

Britton \& Rose (1919-1922) la tratan como Cactus townsendii Britton \& Rose 1922, sin embargo el género no es válido por lo que se respeta el nombre publicado por Vaupel en 1913. Es por esto que es tratado por Hunt (1999), Anderson (2001), Ostolaza et al. (2005) y Hunt et al. (2006) como Melocactus peruvianus Vaupel.

Esta especie se encuentra en estado Vulnerable de acuerdo a Ostolaza (2003), para el caso del Perú. Esta categoría, agrupa a los taxones que enfrentan un riesgo de extinción a mediano plazo, con poblaciones pequeñas o restringidas (IUCN, 2001).

Tribu Browningieae Buxbaum

Plantas arborescentes, arbustivas o columnares; tallos gruesos con costillas, segmentados o no, cubiertos de numerosas espinas; zonas reproductivas no diferenciadas, a veces poco espinosas; flores sésiles, laterales, nocturnas, tubulares, infundibuliformes o hipocrateriformes, actinomorfas; pericarpelo con escamas, pelos, areolas y espinas; frutos carnosos, desnudos o con escamas o espinas; semillas rugosas o ruminadas sin expansiones (Anderson, 2001; Barthlott \& Hunt, 1993).

Neoraimondia Britton \& Rose. Cactaceae. Jahrbücher der Deutschen Kakteen-Gesellschaft 2: 181-182, f. 257-260. 1920.

Planta arbustiva o arborescente. Tallo terete, erguido, 4 a 8 costillas ampliamente separadas, areolas muy desarrolladas, redondas a elongadas, marrones, de crecimiento continuo, con espinas muy grandes. Flor actinomorfa, infundibuliforme, diurna, rosada o blanca, pericarpelo y tubo floral con brácteas a manera de escamas, cerdas y espinas. Fruto globular, con areolas y espinas, semillas numerosas y negras. Distribución: Perú y Bolivia (Hunt et al., 2006).

Neoraimondia arequipensis (Meyen) Backeb. Blätter für Kakteenforschung. 1936. Cereus arequipensis Meyen. Allgemeine

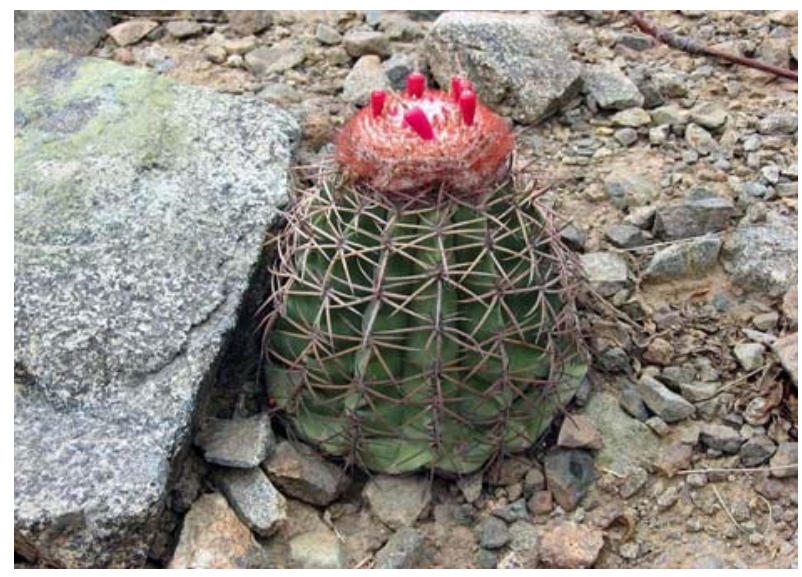

Figura 3. Melocactus peruvianus Vaupel. 

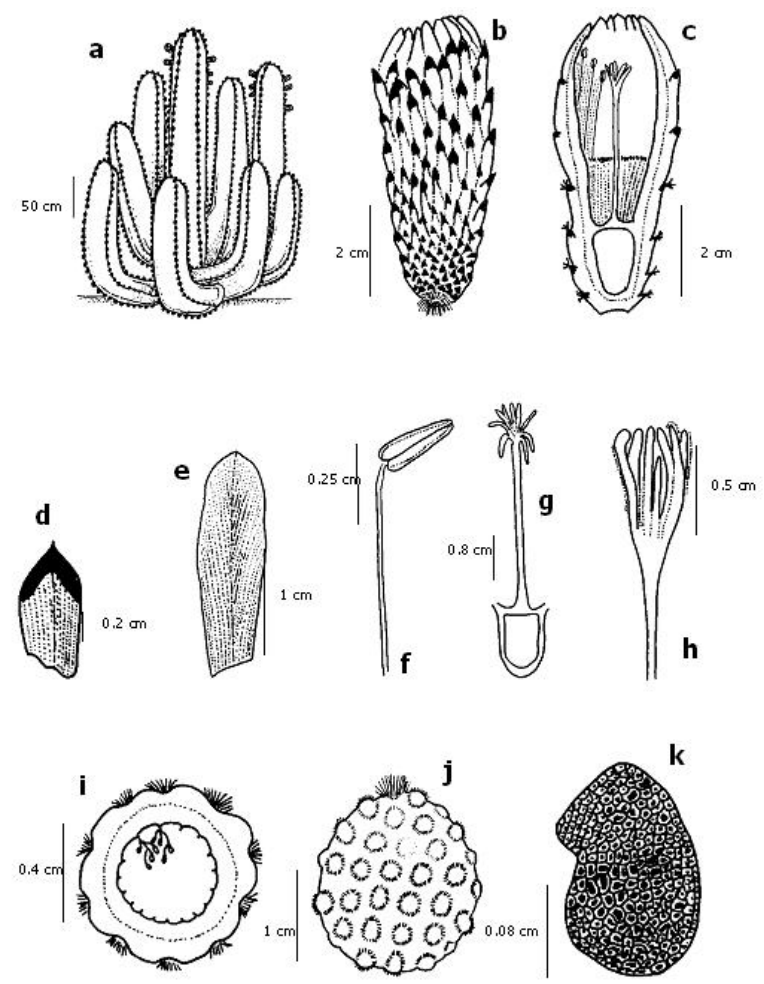

Figura 4. Neoraimondia arequipensis subsp. roseiflora (Werderm. \& Backeb.) Ostolaza. a. Hábito; b. Flor; c. Sección longitudinal de la flor; d. Tépalo sepaloide; e. Tépalo petaloide; f. Estambre; g. Gineceo; h. Estigma; i. Sección transversal del ovario; j. Fruto; k. Semilla.

\section{Gartenzeitung 4:258. 1833.}

Sinónimo: Pilocereus macrostibas Schumann, Cereus macrostibas (Schumann) Berger, Neoraimondia macrostibas (Schumann) Britton \& Rose.

Planta arbustiva, ramificada desde la base; ramas gruesas, columnares, de $10 \mathrm{~m}$ de longitud y $40 \mathrm{~cm}$ de diámetro; 5 a 8 costillas con prominentes areolas de 5 $\mathrm{cm}$ de longitud; número variable de espinas llegando hasta 7, de $25 \mathrm{~cm}$ de longitud; flores blanco verdoso hasta rosado, de $3 \mathrm{~cm}$ de longitud; pericarpelo con pelos cortos y pequeñas espinas; frutos circulares, guindas, de $7 \mathrm{~cm}$ de diámetro, con pericarpo cubierto de areolas circulares y espinas cortas. La especie se distribuye en Piura, Lima, Ica, Arequipa y Tacna entre los 500 y 2500 msnm (Anderson, 2001; Hunt et al., 2006). En la zona de estudio se determinó una subespecie.

Neoraimondia arequipensis subsp. roseiflora (Werderm. \& Backeb.) Ostolaza. Cactaceae Consensus Initiatives 6:9. 1998. Cereus macrostibas var. roseiflorus Werderm. \& Backeb.. Neue Kakteen 74. 1931.

Sinónimo: Neoraimondia roseiflora (Werderm. \& Backeb.) Backeb.
Planta columnar robusta que alcanza de 1.3 a $4 \mathrm{~m}$ de alto, ramificada desde la base, formando grupos de numerosos tallos. Tallo terete de 15 a $20 \mathrm{~cm}$ de diámetro, con 4 a 6 costillas, epidermis visible y verde, con cutícula; areolas grandes de crecimiento continuo, de forma aovada a alargada, de 1.3 a $3 \mathrm{~cm}$ de largo y 2 a 3 cm de diámetro, color marrón oscuro; 6 a 21 espinas por areola, de 6 a $13 \mathrm{~cm}$ de longitud, $0.3 \mathrm{~cm}$ de grosor, de color gris; flores actinomorfas, infundibuliformes, rosadas a rojo grosella, desarrollándose sobre las areolas floríferas, entre 4.7 y $6 \mathrm{~cm}$ de longitud y perianto de 1.7 y 2.2 de diámetro, con aproximadamente 40 a 50 tépalos: sepaloide, lanceolado-apiculado, con el ápice negro, de 0.5 a 1.6 cm de longitud y de 0.3 a $0.5 \mathrm{~cm}$ de ancho, y petaloide ligulado-rotundo, de 1.4 a $2.3 \mathrm{~cm}$ de longitud y de 0.4 a $0.6 \mathrm{~cm}$ de ancho; tubo del receptáculo, de 3.5 a 4.5 cm de longitud y de 1.1 a 1.5 cm de diámetro; cámara nectarial de 1.1 a $1.4 \mathrm{~cm}$ de longitud y de 0.5 a $0.7 \mathrm{~cm}$ de diámetro; androceo con aproximadamente 350 estambres epipétalos, entre 1.1 y $1.8 \mathrm{~cm}$ de longitud, anteras bitécicas entre 0.2 y $0.3 \mathrm{~cm}$ de longitud y 0.1 cm de diámetro, amarillas, filamentos entre 0.9 y 1.5 $\mathrm{cm}$ de longitud y $0.05 \mathrm{~cm}$ de diámetro, color marfil; pericarpelo de 1.3 a $1.8 \mathrm{~cm}$ de longitud y de 1.1 a 1.6 cm de diámetro, cubierto con pequeñas brácteas a manera de escamas, pelos rizados y pequeñas espinas; gineceo sincárpico de 3.9 a $5.4 \mathrm{~cm}$ de longitud, ovario ínfero de 0.5 a $1.3 \mathrm{~cm}$ de longitud y entre 0.4 a $0.8 \mathrm{~cm}$ de diámetro, 10 a 12 carpelos, numerosos óvulos en placentación parietal, estilo de 2 a $2.5 \mathrm{~cm}$ de longitud y de 0.2 a $0.3 \mathrm{~cm}$ de diámetro, estigma lobulado de 0.5 cm de longitud y 0.74 cm de diámetro, 10 a 15 lóbulos color verde; fruto baya, de color rojo a guinda, globular de 3.5 a $4 \mathrm{~cm}$ de diámetro, cubierto de areolas redondas de $0.4 \mathrm{~cm}$ de diámetro con pelos y espinas pequeñas, funículo ariloso blanco rosado desarrollado (Figuras 4 y 5). De acuerdo a lo reportado por Ramírez \& Ceroni (2009) las semillas

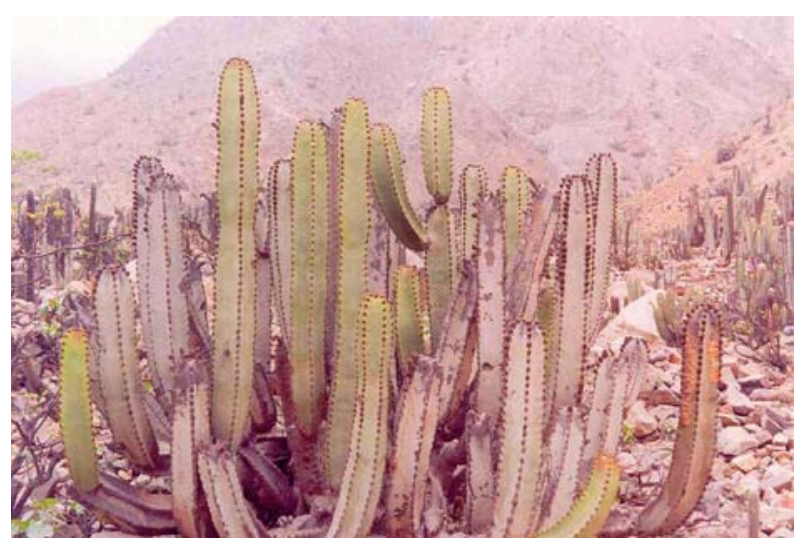

Figura 5. Neoraimondia arequipensis subsp. roseiflora (Werderm. \& Backeb.) Ostolaza. 
son de forma reniforme-ovalada, negras o marrones, numerosas (1000 a 2000), de $1.95 \mathrm{~mm}$ de longitud y $1.28 \mathrm{~mm}$ de diámetro, testa de superficie ruminada y región hilo-micropilar grande y oblicua con respecto al eje mayor de la semilla. Se distribuye en el Perú, en los departamentos de Ancash, Lima e Ica, entre los 500 y 1700 msnm, según los valles, siendo su límite norte el valle del río Fortaleza en Ancash y su límite sur en Pisco, Ica (Hunt et al., 2006; Ceroni et al., 2007).

Material estudiado: LIMA: Cerro Umarcata, Santa Rosa de Quives, febrero de 2004, 1260 msnm, Viviana Castro 115 (Herbario de Sitio Jardín Botánico OVN); Santa Rosa de Quives, abril de 1953, 1300 msnm, R. Ferreyra, 9011 (USM); Paso Huachoc Canta, agosto de 1957, 1000 msnm, P. Hutchinson, 1036 (USM).

Según Anderson (2001) la especie fue descrita originalmente por Meyen en 1833 como Cereus arequipensis y más tarde redescrita por Schumann en 1903 como Pilocereus macrostibas.

En 1920 Britton \& Rose (1919-1922) publican el género Neoraimondia en honor a Antonio Raimondi, basándose en la descripción hecha por Schumann; de este modo la especie es tratada como Neoraimondia macrostibas (Schumann) Britton \& Rose y se caracteriza por sus abultadas areolas, espinas de $25 \mathrm{~cm}$ y flores blancas.

Ostolaza et al., (1985) y Anderson (2001) indican que Backeberg demostró en base a comparaciones de material vegetal y en las descripciones originales, que las especies descritas como Cereus arequipensis, Pilocereus macrostibas y Neoraimondia macrostibas, correspondían a la misma planta; Backeberg la publicó en 1936 como Neoraimondia arequipensis (Meyen) Backeb. La especie presenta dos subespecies que se diferencian por el color de la flor y el número de costillas. Una de estas subespecies ya había sido publicada previamente como Neoraimondia roseiflora (Werderm. \& Backeb.) Backeb. en 1932.

Esta subespecie es tratada por Ostolaza (1996) bajo el nombre de Neoraimondia arequipensis var. roseiflora (Backeb.) Ritter, para tipificar la población cuya variación se encuentra en el color rosado de las flores.

Sin embargo Hunt (1999) la reporta como Neoraimondia arequipensis (Meyen) Backeb., dado que no acepta que las variaciones locales sean suficientes para que se consideren subespecies distintas.

Posteriormente es tratada por Anderson (2001) como Neoraimondia arequipensis (Meyen) Backeb., aunque acepta la existencia de dos subespecies que se diferencia en el color de la flor, número de costillas y la localidad de origen.

Ostolaza et al. (2005) la tratan como Neoraimondia arequipensis subsp. roseiflora (Werderm. \& Backeb.) Ostolaza, la cual se caracteriza por tener como máximo 5 costillas, flores de color rosado a rojo y se distribuye en los valles de Chosica hasta Pisco, lo cual coincide con las características de los ejemplares encontrados en la zona de estudio.

Sin embargo Hunt et al. (2006) consideran a Neoraimondia arequipensis subsp. roseiflora (Werderm. \& Backeb.) Ostolaza, como un sinónimo de Neoraimondia roseiflora (Werderm. \& Backeb.) Backeb., cuya distribución se restringe a Chosica en Lima, que a su vez es un sinónimo de Neoraimondia arequipensis (Meyen) Backeb., cuya distribución es desde Piura hasta Tacna, lo que coincide con lo publicado originalmente por Backeberg en 1936. Esto refuerza la hipótesis que la variación en el color de la flor podría deberse a fenómenos locales y no a la existencia de subespecies.

Esta especie se encuentra en estado de Preocupación Menor de acuerdo a Ostolaza (2003), para el caso del Perú. Esta categoría, agrupa a los taxones que presentan abundancia de individuos y extensa área de ocupación, por lo que se le considera un grupo exitoso (IUCN, 2001).

\section{Literatura citada.}

Anderson E.F. 2001. The Cactus Family. Timber Press, Inc. U.S.A.

Barthlott W. \& Hunt R. 1993. Cactaceae. En: K. Kubitzki (Ed) The families and genera of vascular plants. Vol II: Flowering Plants. Dicotyledons. Springer-Verlag Berlin Heidelberg. Alemania. : 161-196.

Britton N. \& Rose J. 1919-1922. The Cactaceae I, II, III \& IV. Carnegie Institution. U.S.A.

Calderón Moya-Méndez N., Ceroni Stuva A. \& Ostolaza Nano C. 2004. Distribución y estado de conservación del género Haageocereus (Familia Cactaceae) en el departamento de Lima. Perú. Ecología Aplicada. 3 (1 y 2): 17 - 22.

Calderón N., Zappi D., Taylor N. \& Ceroni A. 2007. Taxonomy and conservation of Haageocereus Backeb. (Cactaceae) in Peru. Bradleya. 25: 45-124.

Castro V. 2006. Taxonomía de la familia Cactaceae en el valle de río Chillón, Lima: cerro Umarcata y quebrada Oropel. Tesis para optar el Grado Académico de Magister Scientiae. UNALM.

Ceroni A., Castro V., Calderón N. \& S. Novoa. 2004. Cactario del Jardín Botánico "Octavio Velarde Núñez" de la Universidad Nacional Agraria La Molina. Quepo. Vol. 18. : 34 - 38

Ceroni Stuva A. \& Castro Cepero V. 2006. Los cactus del cerro Umarcata. Lima. Canta. Perú. Revista de Biología Augusto Weberbauer. 9: 15 - 18.

Ceroni A., Castro V., Teixeira V. \& Redolfi I. 2007. Neoraimondia arequipensis subsp. roseiflora (Werderm. \& Backeb.) Ostolaza (Cactaceae): eje de las interacciones en ecosistemas áridos. Ecología Aplicada. 6 (1 y 2): 155 - 168.

Hunt D. 1999. CITES Cactaceae Checklist. Royal Botanic Gardens Kew \& International Organization for Succulent Plant Study. England.

Hunt D., Taylor N. \& Charles G. 2006. The New Cactus Lexicon. DH Books. U.K. 
International Union for Conservation of Nature and Natural Resources (IUCN). 2001. IUCN Red List Categories and Criteria: Version 3.1. IUCN Species Survival Commission. IUCN, Gland, Switzerland and Cambridge, UK.

Lozada-Castillo J. 2009. Cactáceas de la provincia de Tumbes, Tumbes, Perú. Quepo. 23: 70-79.

Novoa S., Ceroni A. \& Arellano C. 2005a. Contribución al conocimiento de la fenología del cactus Neoraimondia arequipensis subsp. roseiflora (Werderm. \& Backeb.) Ostolaza (Cactaceae) en el valle del río Chillón, LimaPerú. Ecología Aplicada. 4 (1 y 2): 35-40.

Novoa S., Redolfi I. \& Ceroni A. 2005b. Patrón de actividad diario de la hormiga Camponotus sp. en los botones florales del cactus Neoraimondia arequipensis subsp. roseiflora (Werderm. \& Backeb.) Ostolaza. Ecología Aplicada. 4 (1 y 2): 77-81.

Novoa S., Redolfi I., Ceroni A. \& Arellano C. 2005c. El forrajeo de la hormiga Camponotus sp. en los botones florales del cactus Neoraimondia arequipensis subsp. roseiflora (Werderm. \& Backeb.) Ostolaza (Cactaceae). Ecología Aplicada. 4 (1 y 2): 83-90.

Ostolaza C. 1996. A closer look at the conservation status of Cacti in the vicinity of Lima, Peru. British Cactus and Succulent Journal. 14(4): 158-174.

Ostolaza C. 2003. Categorización y conservación de Cactáceas Peruanas. Zonas Aridas. 7: 194-205.
Ostolaza C. 2007. Cactus del norte de Ancash, Perú. Quepo. 21: 59-73.

Ostolaza C., Mitich L. \& King J. 1985. Neoraimondia arequipensis var. roseiflora (Werd. \& Backbg.) Rauh. Cactus \& Succulent Journal. 57(1): 60-64.

Ostolaza C., Ceroni A., Calderón N. \& Novoa S. 2005. The Huaura Valley, Lima, Peru - revisited. British Cactus and Succulent Journal. 23(1): 25-33.

Ramírez N. \& Ceroni A. 2009. Caracterización morfológica de semillas de cactáceas del Cerro Umarcata, Canta, Lima, Perú. Quepo. 23: 90-98.

Taylor N.P. \& Zappi D.C. 2004. The Cacti of eastern Brazil. Royal Botanic Gardens of Kew. Richmond, U.K.

Teixeira V., Castro V., Ceroni A. \& Eyzaguirre R. 2004. Diversidad y densidad de la comunidad de cactáceas en el valle del río Chillón: cerro Umarcata y quebrada Orobel y su relación con los factores edáficos. Ecología Aplicada. 3 (1 y 2): 1-8.

Wallace R.S. \& Gibson A.C. 2002. Evolution and Systematics: 1-21. In: Nobel, P.S. Cacti: Biology and Uses. Univ. California Press. Berkeley.

Weberbauer A. 1945. El mundo vegetal de los Andes Peruanos: Estudio fitogeográfico. 2da Edición. Estación Experimental Agrícola de La Molina. Dirección de Agricultura. Ministerio de Agricultura. Lima.

1 Jardín Botánico “Octavio Velarde Núñez”. Departamento Académico de Biología, Universidad Nacional Agraria La Molina. Av. La Molina s/n, Lima 12, Perú. Apartado postal: 12-056, Perú. Correo electrónico: vcastro@lamolina.edu.pe.

2 Jardín Botánico “Octavio Velarde Núñez”. Departamento Académico de Biología, Universidad Nacional Agraria La Molina. Av. La Molina s/n, Lima 12, Perú. Apartado postal: 12-056, Perú. Correo electrónico: aceroni@lamolina.edu.pe. 